Technological University Dublin

ARROW@TU Dublin

\title{
An Equivalent Circuit for Corona Discharges Caused by a Point to Plane Arrangement at ac, dc and Combined Voltages
}

Tobias Dezenzo

Darmstadt University of Applied Sciences, Germany

Thomas Betz

Darmstadt University of Applied Sciences, Germany

Andreas Schwarzbacher

Technological University Dublin, andreas.schwarzbacher@tudublin.ie

Follow this and additional works at: https://arrow.tudublin.ie/engschececon

Part of the Electrical and Computer Engineering Commons

\section{Recommended Citation}

Dezenzo, T., Betz, T. \& Schwarzbacher, A. (2017). An Equivalent Circuit for Corona Discharges Caused by a Point to Plane Arrangement at ac, dc and Combined Voltages. Conference: 2017 IEEE Conference on Electrical Insulation and Dielectric Phenomenon (CEIDP)Cancun, Mexico, Oct. 21-24, 2018. doi:10.1109/ CEIDP.2017.8257456

This Conference Paper is brought to you for free and open access by the School of Electrical and Electronic Engineering at ARROW@TU Dublin. It has been accepted for inclusion in Conference papers by an authorized administrator of ARROW@TU Dublin. For more information, please contact arrow.admin@tudublin.ie, aisling.coyne@tudublin.ie,gerard.connolly@tudublin.ie.

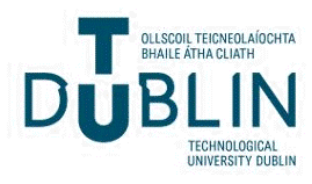




\title{
An equivalent circuit for corona discharges caused by a point to plane arrangement at ac, dc and combined voltages
}

\author{
Tobias Dezenzo ${ }^{1,2}$, Thomas Betz ${ }^{1}$, Andreas Schwarzbacher ${ }^{2}$ \\ ${ }^{1}$ Darmstadt University of Applied Sciences, Birkenweg 8, 64295 Germany \\ ${ }^{2}$ Dublin Institute of Technology, Kevin Street, Dublin 8
}

\begin{abstract}
In this paper a new two stage equivalent circuit for corona discharges is introduced. Its aim is to fill the gap between complexity of the circuit and accurate representation of the physical properties, which underlay a corona discharge. The first stage represents the voltage distribution and the occurrence of a discharge with respect to the applied voltage. The second stage models the physical discharge behavior like ionization, number of electrons, positive and negative ions as well as their interaction. The proposed circuit is able to model the different modes of corona discharges, which is experimentally verified. The simulation results are in good agreement with the measured results but cannot compete with simulations based on solving continuity equations. Nevertheless, due to the solely basement on network theory, it provides an easy access to the occurring physical effects during a corona discharge for all electrical engineers.
\end{abstract}

\section{INTRODUCTION}

The use of equivalent circuits is a common technique in the different fields of electrical engineering. It is used to reduce the complexity of physical problems by introducing electrical components that have one specific behavior. The big advantage is the common understanding of the current-voltage relations of the components used. The disadvantage lays in the proportionality between complexity of the circuit and the accuracy in representing the real physical mechanisms.

For partial discharges (PD) different equivalent circuits depending upon the type of PD have been introduced e.g. [1, 2]. The main types are corona discharge, gliding or dielectric barrier discharge and internal partial discharges. The related equivalent circuits have in common that there complexity is limited, which makes them easy to understand. This reduced complexity earned them criticism, especially for the famous abc-equivalent circuit of internal PD [3].

The present equivalent circuit of corona discharges is also not able to reflect the physical occurrences during a partial discharge but it can explain the position of the discharge related to the waveform of the applied voltage. A more precise equivalent circuit has for example been introduced for positive glow corona in a coaxial cylinder geometry [4]. However, no circuit exists that covers the whole range of corona discharge phenomenon.

Therefore, a new two stage equivalent circuit for the representation of corona discharges is introduced in this paper.
The basic idea behind the two stage circuit is to overcome the discrepancy between voltage distribution and physical problems by splitting the equivalent circuit into two parts, where each part is responsible for one aspect.

\section{THE EQUIVALENT CIRCUIT}

\section{A. First stage}

The first stage of the circuit, which is shown in Fig. 1 is attributed to the voltage distribution. The source is of alternating voltage type but can be interchanged or superimposed with either a negative or a positive direct voltage source. One essential limitation for the ac source is its frequency, which must be small compared to the clearing times of the generated particles. The diodes $D_{1}$ and $D_{2}$ are used to differentiate between positive and negative polarity as the underlying physical discharge processes are different. The parallel connections $R_{1}, C_{1}$ and $R_{3}, C_{2}$ are used to model the buildup of the electric field stress and therefore, partially determine the time between discharges. Each part contains a variable sphere-gap $S g$, which has two functions. The first is attributed to the presence of a starting electron, otherwise the discharge would always start at the same voltage level and the second function is that it serves as a place holder for the second stage of the equivalent circuit. The series resistors $R_{2}$ and $R_{4}$ are required to model the background current, which is assumed to be of constant type.

\section{B. Second stage}

The second stage is used to model the different discharge mechanisms. These are Trichel pulses and pulseless glow

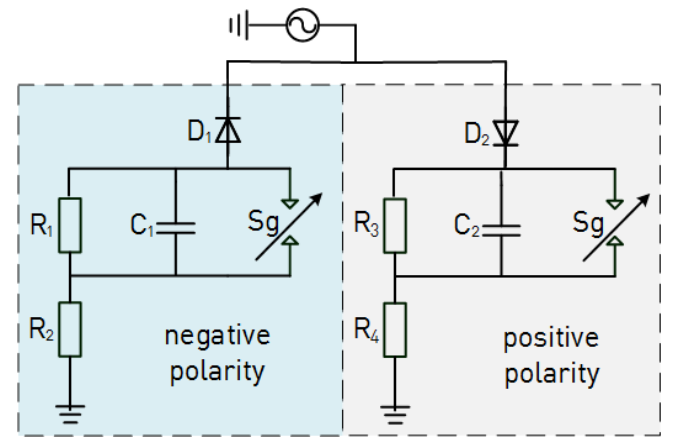

Fig. 1. Equivalent circuit for the voltage distribution of a point-to-plane arrangement. 


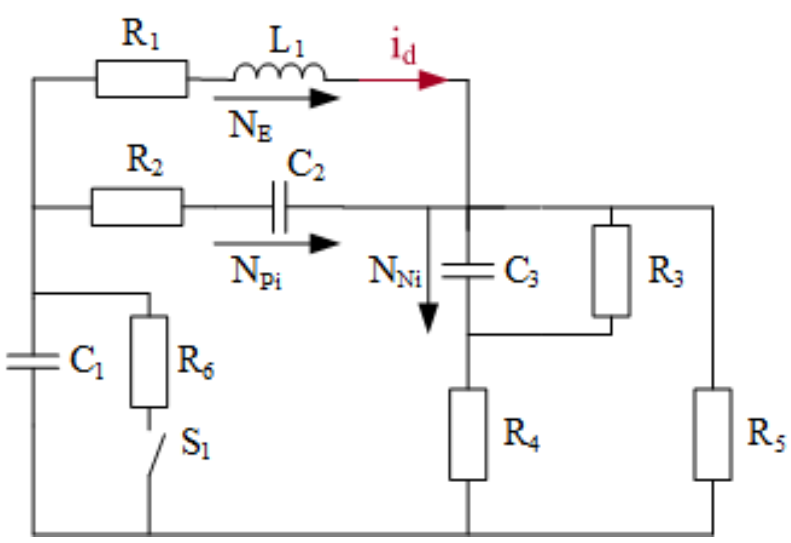

Fig. 2. Equivalent circuit for negative polarity Trichel pulses and pulseless glow.

(negative polarity) as well as onset streamers, burst pulses, positive glow and pre-breakdown streamers (positive polarity). Negative streamers have not been modeled.

The equivalent circuit for negative polarity is shown in Fig. 2. In the negative polarity case the first part is formed by the capacitor $C_{1}$, the resistor $R_{6}$ and the switch $S_{1}$, which represent the electrical field and therefore the ionization, which can be choked off by closing the switch. The second part is attributed to the electron and positive ion generation and their interaction. It is model by the parallel connection of the series connections $R_{1}, L_{1}$ and $R_{2} C_{2}$. The voltage drops at the energy storages represent the amount of electrons $N_{E}$ and positive ions $N_{P i}$ in the ionization layer. The discharge current $i_{d}$ is measured in the inductive branch. The resistors $R_{3}, R_{4}, R_{5}$, and the capacitance $C_{3}$ are used to model the behavior of the negative ions. Their number $N_{N i}$ is related to the voltage drop across the capacitor $C_{3}$. This model is intended for Trichel pulses and the pulseless glow.

The switch $S_{1}$ and the resistor $R_{6}$ have been omitted for the positive voltage polarity (Fig. 3). In the second part of the circuit the structure is identical as the one used in the negative case. However, the attributes of the elements have changed. The voltage drop at the inductance $L_{1}$ now represents the number of electrons $N_{E h}$ and positive ions $N_{P i h}$ in the streamer head. The third part of the circuit is related to the movement of the streamer head into the gap. The resistor $R_{3}$ represents the velocity of the streamer $v$, whose advancement into the air gap

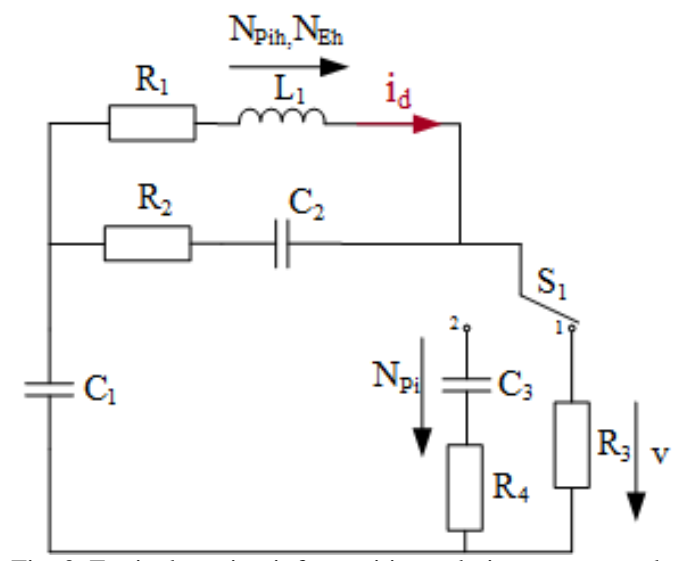

Fig. 3. Equivalent circuit for positive polarity streamer pulses. can be stopped by turning the switch $S_{1}$ into position 2 . The now active part of the circuit is attributed to the formation of a residual positive space charge at the anode $N_{P i}$. Negative ions have been neglected.

A second circuit for positive corona is required as the burst pulse and the positive glow processes differ from the streamer pulse mechanism due to their radial spread across the anode [5]. The circuit structure is similar to the one depicted in Fig. 2. Only the resistors $R_{3}, R_{5}$ and $R_{6}$ as well as the switch $S_{1}$ have been omitted.

\section{MEASURING SETUP}

For the verification of the functionality of the proposed circuits measurements using a point-to-plane arrangement have been carried out. All measurements have been performed with a gap spacing of $30 \mathrm{~mm}$ and a point electrode of about $0.5 \mathrm{~mm}$ radius. The insulating gas was air at atmospheric pressure at a temperature of $20^{\circ} \mathrm{C}$.

The dc voltage is obtained due to a half wave rectification of the applied ac voltage. Even though, a smoothing capacitor $C_{S}$ of $20 \mathrm{nF}$ has been used the dc voltage contains a small ripple of about $0.1 \%$ due to a constant voltage measurement. The discharge current is measured in series to the rogowski-shaped plate. A HBM Gen7t with a HBM GN110 probe in combination with a $1 \mathrm{k} \Omega$ shunt resistor is used to measure the discharge current. The specifications of the measuring system are: 14 bit resolution, $25 \mathrm{MHz}$ bandwidth and $100 \mathrm{MS}$ / s.

Tests have been conducted from corona onset just before breakdown for both polarities.

\section{RESULTS AND DISCUSSION}

\section{A. Negative corona}

The results of a measured and a simulated Trichel pulse (circuit according to Fig. 2) are depicted in Fig. 4. The corresponding particle behavior of the electrons, positive ions and negative ions for the rise of the pulse until approximately half pulse decay is shown in Fig. 5. A good agreement between measured and simulated pulse during the rise of the pulse as well as in the decay phase is visible. There are discrepancies in the vicinity of the maximum of the pulse. These discrepancies are attributed to two factors. The first

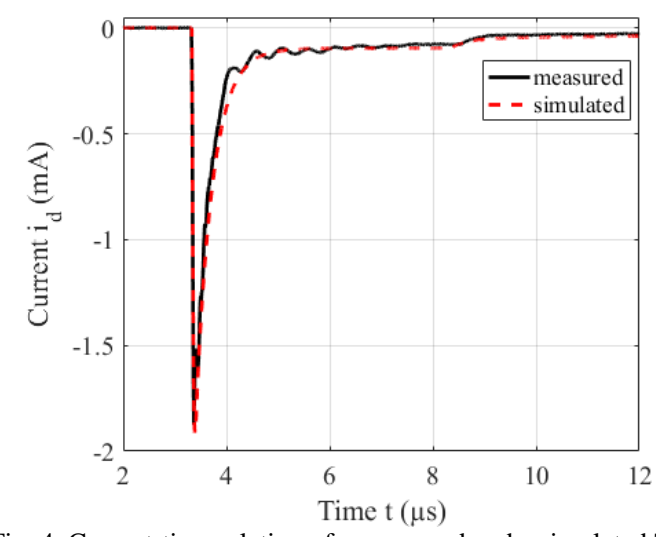

Fig. 4. Current-time relation of a measured and a simulated Trichel pulse. 
factor is the improper adjustment of the different component values in the equivalent circuit and the second are the measuring uncertainties caused by the limited bandwidth and the resolution. Nevertheless, the general pulse shape is represented with good accuracy.

An analysis of the particle behavior reveals an exponential increase of the number of electrons as well as the number of positive ions and negative ions. The underlying physical process can be explained as follows: If a starting electron is available an electron avalanche will start, where electron multiplication via impact ionization takes places. Therefore, electrons and positive ions are exponentially increasing. If a positive space charge in form of positive ions has built up in the ionization region the avalanche is choked off and the number of electrons due to avalanche ionization decreases. The positive ions will then wander towards the cathode and will release secondary electrons due to cathode bombardment, which is represented by the positive amount of electrons in the ionization region (after about $3.4 \mu \mathrm{s}$ ). These processes are all caused by the damped resonant behavior of $L_{1}$ and $C_{2}$ in the equivalent circuit. Due to the increasing amount of negative ions this process is continuously weakened until at about 8.5 $\mu$ s (Fig. 4), where the ionization is completely choked off. This process is realized by closing switch $S_{1}$. A new pulse can first start if the negative ions have moved sufficiently far away [6].

If a series of Trichel pulses is desired than a chain of circuits can be used, where the previous circuit will always determine the space charge conditions for the next circuit. Each circuit itself can therefore be treated as if its pulse would occur in a space charge free environment. A resulting pulse series, which undergoes the transition into the pulseless glow is shown in Fig. 6. The glow transition has also been modeled using the circuit according to Fig. 2 by not using the switch $S_{1}$, as the negative ions do not choke off the ionization in this discharge
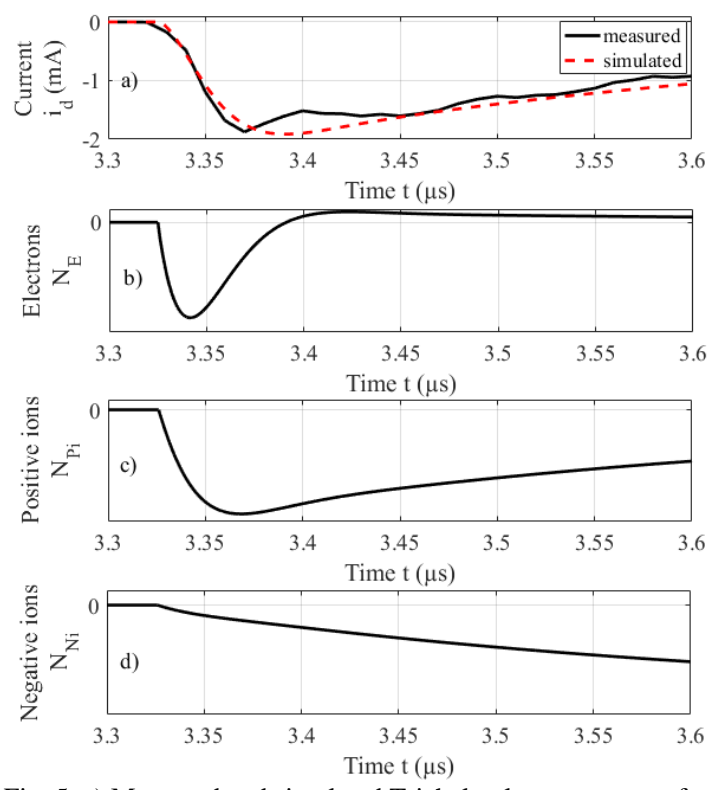

Fig. 5. a) Measured and simulated Trichel pulse current as a function of time. Associated number of b) electrons, c) positive ions and d) negative ions as a function of time.

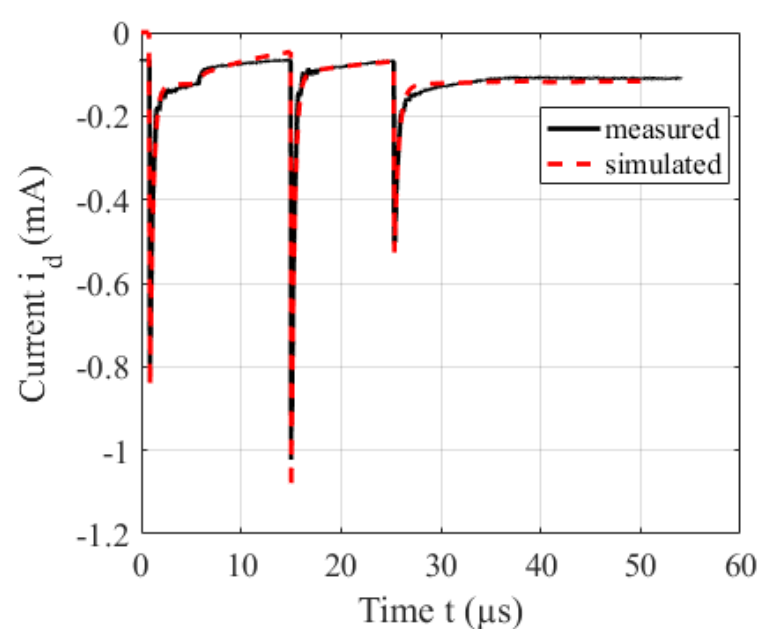

Fig. 6. Measured and simulated series of Trichel pulses including the transition to the pulseless glow.

mode [7]. As the corona current in the glow regime is proportional to the applied voltage and therefore to the field strength it can be increased by adjusting the voltage of capacitor $\mathrm{C}_{1}$ in the equivalent circuit.

\section{B. Positive Corona}

Fig. 7 shows the time dependency of the current of a measured and a simulated streamer pulse. During the rise of the pulse and the pulse decay the agreement is good, whereas a small deviation is found during the plateau phase of the pulse. A more detailed representation of the current pulse and the particle behavior is shown in Fig. 8.

During the rise of the pulse the streamer advances from the anode into the low field region of the gap, which leads to a reduction of the streamers velocity [8] and an increase of electrons and positive ions in the streamer head. In the plateau region of the current pulse the streamer velocity stays constant [9] as does the amount of electrons and positive ions. The former processes are caused by the damped resonant behavior of $L_{1}$ and $C_{2}$. Current decay is initiated after the streamer stops advancing. This is realized by turning switch $S_{1}$ from position 1 to position 2. The electrons then travel into the high field region of the anode, where a positive space charge is formed due to impact ionization. The positive space charge reduces

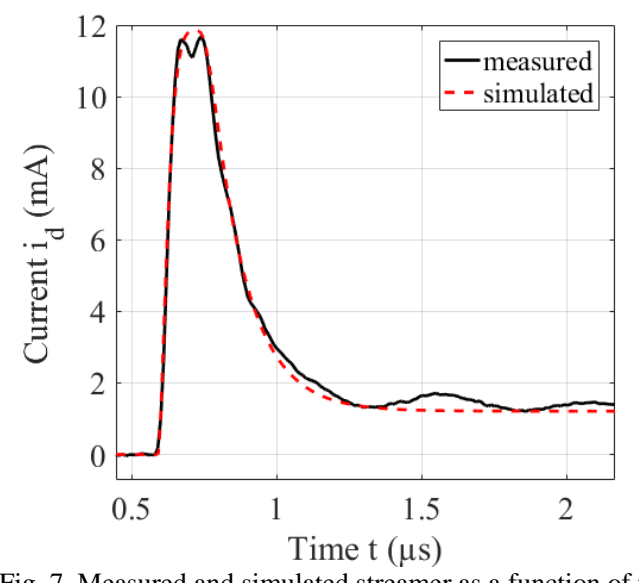

Fig. 7. Measured and simulated streamer as a function of time. 
the field in the vicinity of the anode. The presence of the positive space charge favors burst pulse activity as they prevent axial discharge spread until they have moved sufficiently far away. A measured and a simulated burst pulse series as well as the corresponding particle behavior is shown in Fig. 9. It is evident that the number of electrons and positive ions increase during the rise of the pulse because of impact ionization. The electrons are than rapidly absorbed by the anode, whereas the positive ions will wander slowly towards the cathode. After the electrons have vanished new seed electrons are required to cause an avalanche and therefore another pulse. The negative amount of electrons represents the secondary electron generation, which is assumed to largely take place outside of the ionization region. The photoionization is larger in the maximum of the current pulse as in the minimum, as has been shown by [10]. Therefore, more electrons will be arriving at the ionization region shortly after the pulses maximum but due to the presence of the positive ions the field is too weak to cause another avalanche. Burst pulse activity therefore ceases if the positive ions are still too close to the ionization region, when the photoionization is reduced.

If the field is further enhanced burst pulse activity increases until the pulsating glow regime is reached. The pulsating glow is basically a never ending burst pulse [11]. Therefore, an additional modeling is not required. The same holds true for breakdown streamers, which have the same waveform as the onset streamers [12].

\section{CONCLUSION}

A novel two stage equivalent circuit for the simulation and explanation of corona discharges has been introduced. The first stage is required to relate the discharge occurrence to the
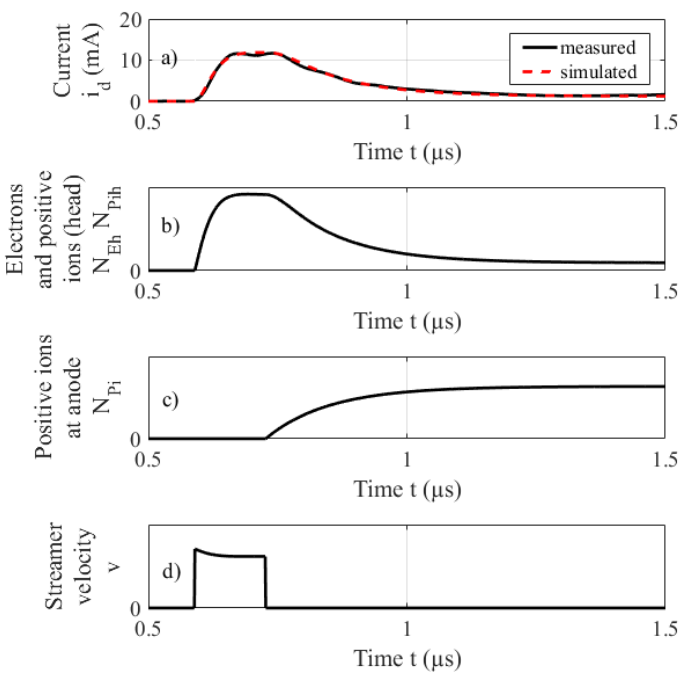

Fig. 8. a) Measured and simulated streamer current as a function of time. Associated number of $b$ ) electrons and positive ions in the streamer head, c) positive ions at the anode and d) streamer velocity as a function of time.
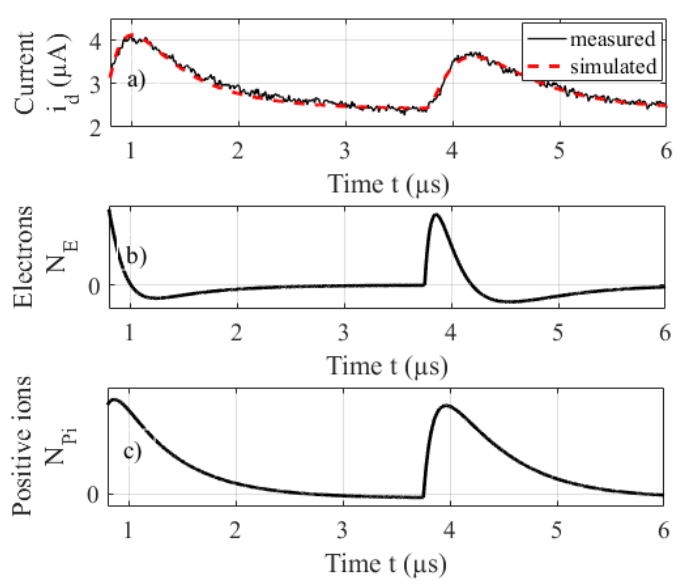

Fig. 9 a) Measured and simulated burst pulse current as a function of time. Associated number of b) electrons and c) positive ions as a function of time.

applied voltage and differentiate between positive and negative corona. The second stage models the physical mechanisms behind the discharge depending on the voltage polarity and the discharge mode.

By using this two stage approach the discrepancy between accuracy in physical property representation and complexity of the required circuit could be removed. It is therefore for the first time possible to explain the physical mechanisms of corona discharges using only a simplified theoretical network approach.

\section{REFERENCES}

[1] D. Koenig and Y. Narayana Rao, Partial discharges in electrical power apparatus. Berlin: VDE-Verlag, 1993.

[2] F. H. Kreuger, Discharge Detection in High Voltage Equipment. London: Temple press books ltd, 1964.

[3] E. Lemke, "A critical review of partial-discharge models," IEEE Electrical Insulation Magazine, vol. 28, no. 6, pp. 11-16, 2012.

[4] R. S. Sigmond, "The oscillations of the positive glow corona," $J$. Phys. IV France, vol. 07, no. C4, pp. C4-383-C4-395, 1997.

[5] G. W. Trichel, "The mechanism of the positive point-to-plane corona in air at atmospheric pressure," Phys. Rev, vol. 55, no. 4, pp. 382390, 1939.

[6] W. L. Lama and C. F. Gallo, "Systematic study of the electrical characteristics of the "Trichel" current pulses from negative needle-to-plane coronas," Journal of Applied Physics, vol. 45, no. 1, pp. 103-113, 1974.

[7] T. N. Giao and J. B. Jordan, "Trichel streamers and their transition into the pulseless glow discharge,” Journal of Applied Physics, vol. 41, no. 10, pp. 3991-3999, 1970.

[8] M. R. Amin, "Fast time analysis of intermittent point-to-plane corona in air. II. the positive pre-onset streamer corona,” Journal of Applied Physics, vol. 25, no. 3, pp. 358-363, 1954.

[9] A. A. Kulikovsky, "Production of chemically active species in the air by a single positive streamer in a nonuniform field," IEEE Transactions on Plasma Science, vol. 25, no. 3, pp. 439-446, 1997.

[10] L. Liu and M. Becerra, "On the transition from stable positive glow corona to streamers,” Journal of Physics D: Applied Physics, vol. 49, no. 22, p. 225202, 2016.

[11] M. R. Amin, "Fast time analysis of intermittent point-to-plane corona in air I. the positive point burst pulse corona," Journal of Applied Physics, vol. 25, no. 2, pp. 210-216, 1954.

[12] N. G. Trinh, "Partial discharge XIX: discharge in air part I: physical mechanisms," IEEE Electrical Insulation Magazine, vol. 11, no. 2, pp. 23-29, 1995. 